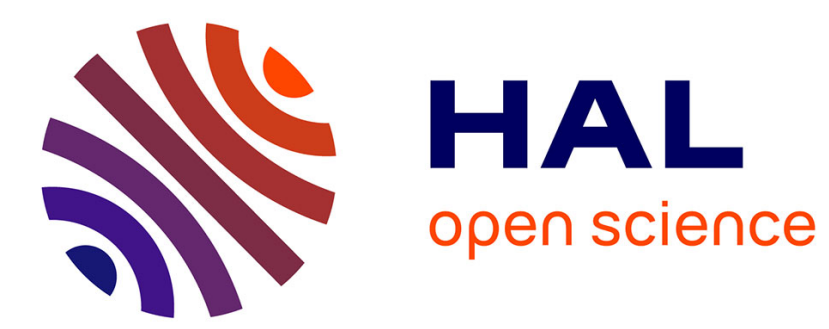

\title{
COMPARISON OF TENDON TENSIONS ESTIMATED FROM TWO BIOMECHANICAL MODELS OF THE THUMB
}

Laurent Vigouroux, Mathieu Domalain, Eric Berton

\section{- To cite this version:}

Laurent Vigouroux, Mathieu Domalain, Eric Berton. COMPARISON OF TENDON TENSIONS ESTIMATED FROM TWO BIOMECHANICAL MODELS OF THE THUMB. Journal of Biomechanics, 2009. hal-03390080

\author{
HAL Id: hal-03390080 \\ https://hal.science/hal-03390080
}

Submitted on 21 Oct 2021

HAL is a multi-disciplinary open access archive for the deposit and dissemination of scientific research documents, whether they are published or not. The documents may come from teaching and research institutions in France or abroad, or from public or private research centers.
L'archive ouverte pluridisciplinaire $\mathbf{H A L}$, est destinée au dépôt et à la diffusion de documents scientifiques de niveau recherche, publiés ou non, émanant des établissements d'enseignement et de recherche français ou étrangers, des laboratoires publics ou privés. 
Journal of biomechanics: Short Communication

1651 words (from the Introduction through the end of discussion)

\section{COMPARISON OF TENDON TENSIONS ESTIMATED FROM TWO BIOMECHANICAL MODELS OF THE THUMB}

Laurent VIGOUROUX $^{1}$; Mathieu DOMALAIN ${ }^{1}$; Eric BERTON ${ }^{1}$

${ }^{1}$ Movement Sciences Institute, Etienne Jules Marey, UMR 6233, Mediterranean University, Marseille, France.

Corresponding author:

Laurent VIGOUROUX

Address: $\quad$ U.M.R. 6233 Institut des Sciences du Mouvement, Faculté des Sciences du Sport, Case postale 910, 163, avenue de Luminy F-13288 Marseille Cedex 09 France

Phone: $\quad+33(0) 491170422$

Fax: $\quad+33(0) 491172252$

Email: $\quad$ laurent.vigouroux@univmed.fr

Keywords: Biomechanical model, thumb, muscle tendon tension; pulp and key pinch, hand 


\section{Abstract (208 words):}

Despite the paramount function of the thumb in daily life, thumb biomechanical models have been little developed and studied. Moreover, only two studies provided quantitative anthropometric data of tendon moment arms. To investigate thumb tendon tensions, biomechanicians and clinicians have to know the performances and the limits of these two data sets. The aim of this study was thus to compare the results of these two models and evaluate their performances in regard to prior electromyographic measurements (EMG). Thumb posture was recorded during the classical key pinch and pulp pinch grips. Various fingertip forces applied at the distal segment were simulated in a range including extension, adduction, flexion, abduction. Input data of thumb postures and fingertip forces were used to compute tendon tensions with both models. Tendon tensions obtained using these two models were then compared and correlated to EMG measurements provided in the literature.

The results showed that both model predicted relevant muscle coordination for five of the nine muscles modelled. Opponent and abductor longus muscle coordinations were badly estimated by both models. Each model was sensible to kinematic errors but not in same proportion. This study pointed out the advantages/limits of the two models in order to use them more appropriately for clinical and/or research purposes. 


\section{Introduction}

To improve hand pathology treatments and surgical techniques, surgeons and clinicians need biomechanical models to know the tensions exerted in the muscle tendons (An et al., 1983; Harding et al., 1993). Despite the primarily importance of thumb functions in daily life, thumb modelling has been less studied and used in the literature (Valero-Cuevas et al., 2003) than the other fingers (Sancho-Bru et al., 2001; Vigouroux et al., 2008). This deficiency is particularly due to the difficult kinematic analysis of the trapezio-metacarpal joint (TMC) where the proximal segment (trapezium bone) is hidden by muscles/soft tissues and is hardly identifiable (Hollister et al., 1992).

Additionally, only Smutz et al. (1998) and Chao et al. (1989) provided usable anthropometric data of tendon moment arms for thumb modelling. Because of the different measurement techniques (tendon excursion vs geometric method) and the use of different specimens, discordances between the two sets of anthropometric data are expected. Moreover, mechanical equilibrium resolution also differs as the moment arms were provided in different reference systems (corresponding to different kinematic analysis methods).

The performances of the two models (Smutz'model, SM vs Chao'model, CM) have never been evaluated or compared in the literature. That leads to improper use of these models without ensure reliable results. The aim of this study was thus to establish recommendations for users of thumb models by comparing the results of the two existing models starting from identical input data.

\section{Material and Methods}

The thumb was modelled as 4 rigid linked-segments (Buchholz, 1992): the proximal and distal phalanges, the metacarpal bone and the trapezium bone. The segments were articulated around three frictionless joints (Fig. 1). The Inter-Phalangeal (IP) joint was 
modelled as a hinge joint with one degree of freedom (flexion/extension). The metacarpophalangeal joint (MP) and the TMC were considered as universal joints with two DoFs in flexion/extension (MPf and TMCf) and in adduction/abduction (MPa and TMCa). The muscles and tendons included in our biomechanical model to actuate the five degrees of freedom are summarized in Table 1.

\subsection{Posture data}

To obtain common input data of thumb postures, six participants (mean age: $28.5 \pm 4.6$ yr, height: $180 \pm 4.2 \mathrm{~cm}$, mass: $77.6 \pm 5.2 \mathrm{~kg}$, hand length: $19.7 \pm 0.6 \mathrm{~cm})$ were asked to adopt an index-thumb "pulp pinch" while gripping a $5.5 \mathrm{~cm}$ width cylinder and a "key pinch" while gripping a key (Valero-Cuevas et al., 2003). The 3D positioning of the thumb segments was recorded by six cameras (Vicon 624 Motion System, Oxford Metrics, England). Three spherical micro-reflective markers ( $4 \mathrm{~mm}$ diameter) were fixed on the dorsal side of each segment. Three markers, placed on the metacarpal bones were used to define the dorsal hand plane reference system ( $\left.\mathscr{R}_{\mathrm{dhp}}\right)$. Starting from the 3D positioning of the segments, joint angles were computed for each model.

For both models, IP and MP joint angles were defined as rotation between distal and proximal segments using reference systems placed on the metacarpal bone $\left(\mathcal{R}_{\mathrm{mc}}\right)$, the proximal phalanx $\left(\mathscr{R}_{\mathrm{pp}}\right)$ and the distal phalanx ( $\left.\mathscr{R}_{\mathrm{dp}}\right)$ (Fig. 1). For SM, TMC angles were defined as the angle between the thumb metacarpal $\left(\mathscr{R}_{\mathrm{mc}}\right)$ and $\mathbb{R}_{\mathrm{dhp}}$. TMCa corresponded to rotation around the $\mathbb{R}_{\mathrm{dhp}} \mathrm{z}$ axis while TMCf was a motion around the $\mathcal{R}_{\mathrm{dhp}} \mathrm{y}$ axis. With SM, adduction and flexion have positive values. For SM, the key pinch was considered as the $0^{\circ}$ position for $\mathrm{TMCa}$ and TMCf (Smutz et al., 1998). For CM, the TMC joint angles were defined as the rotation between thumb metacarpal $\left(\mathscr{R}_{\mathrm{mc}}\right)$ and the trapezium bone reference system $\left(\mathscr{R}_{\mathrm{t}}\right)$. The positioning of $\mathscr{R}_{\mathrm{t}}$ was determined from Chao et al. (1989) who reported that $\mathbb{R}_{\mathrm{t}}$ is rotated by 
$46^{\circ}$ of flexion, $35^{\circ}$ of abduction and $82^{\circ}$ of supination with respect to $R_{\text {dhp }}$. Angles were extracted from the rotation matrix using the Z, Y, X, Euler's sequence (i.e flexion, abduction, supination) with fixed axes situated on the proximal segment (Cooney et al., 1981; Chao et al., 1989). With CM, abduction and flexion have positive values.

\subsection{Simulated fingertip force}

Fingertip force was simulated as $1 \mathrm{~N}$ intensity. Eight directions of fingertip force, varying in $45^{\circ}$ step (dorsal, dorsal-lateral, lateral, lateral-palmar, palmar, palmar-medial, medial, medial-dorsal), were studied (Fig. 1). These forces were applied at half the length of the distal phalanx. The simulated fingertip forces and the posture data were used as input data for the external joint moments calculation and the tendon moment arms determination. For TMC, external force moments were computed in $\mathbb{R}_{\mathrm{dhp}}$ for SM and in $\mathbb{R}_{\mathrm{t}}$ for CM. Tendon tensions of the nine muscles were estimated for each of the eight simulated fingertip forces and for the two postures. Details of the computing method are presented in Supplementary Material. Mean results of the six subjects were considered.

\subsection{Model comparison}

For each muscle, results provided by SM and CM were compared by computing the mean absolute difference between the two models across the conditions (posture and force direction). To evaluate the agreement of model results with electromyography (EMG), we used the experimental results of Valero-Cuevas et al. (2003). These authors measured the EMG activity of the nine thumb muscles during dorsal, lateral, palmar and medial forces in key and pulp pinches. We used these results to define the activity of each muscle at four different levels (null, little, median, high). These EMG classifications are presented together with the results of this current study. A non-parametric correlation of Spearman was performed for each muscle to identify a correlation between each model and EMG data. A $p<0.05$ was considered as a significant correlation. To evaluate the sensibility of each model 
to kinematics errors, the tendon tensions were also computed with $\mathrm{a}+5^{\circ}$ and $-5^{\circ}$ error localisation of $\mathscr{R}_{\text {dhp }}$ axes. Mean differences between original results and results including error were computed for each model and each muscle.

\section{Results and Discussion}

The results (Figure 2 and 3) showed that outputs of the two models presented some similarities (FPL, FPB, APB, ADPT and EPB). Moreover, the correlation with EMG results confirmed the muscle coordination estimated by both models in FPL, FPB, APB, EPL and EPB (Table 2). The EMG results of Kaufman et al., (1999) (not presented in the current study) also validated the muscle implication for FPL, FPB, APB and EPL, in key pinch for both models. One of the most important identified limits of both models is that neither was able to predict reliable tendon tension in OPP and APL muscles. Concerning adductor muscles, since ADPO and ADPT were not distinguished in EMG measurements, we correlated the EMG measurements with the sum of the two parts. The significant correlation showed that SM was more appropriate to predict adductor coordination than CM. For EPL muscle, both models were correlated to EMG. However, SM predicted higher forces than CM which results in excessive tendon tensions.

The disagreement in tendon tensions estimates between CM and SM are due in part to the anthropometric measurements which may have varied between the two studies. For illustration, Fig. 4 presents the moment arms at TMC joint during key pinch with both SM and CM models expressed in Smutz et al. (1998) reference system.

Also, each anthropometric set have to be employed with a high attention to the kinematics method recommended by the authors (i.e. TMC angles and moments were identified in $\mathbb{R}_{\mathrm{t}}$ for

CM and in $\mathscr{R}_{\text {dhp }}$ for SM). The one used in SM ( $\left.\mathscr{R}_{\text {dhp }}\right)$ does not represent accurately the functional anatomy of TMC joint as the metacarpal bone is articulated with the trapezium 
bone. On the other hand, the localisation of $\mathscr{R}_{\mathrm{t}}$ using external markers with CM model, includes additional errors as this location is based on non-personalized anthropometric data. This point is highly susceptible to change tendon tensions as the third rotation axis (supination) of TMC joint is not considered as free and thus is not equilibrated by muscle action. Since the supination axes are differently oriented $\left(R_{\mathrm{t}} v_{S} \mathbb{R}_{\mathrm{dhp}} \mathrm{X}\right.$ axis), the "nonequilibrate" part of external force moment is different according to the two models. This implicates a different report of the moments on the other rotation axes. Moreover, the sensibility analysis showed that SM is more subject to kinematics error measurement than CM particularly for ADPO, APL, EPL and EPB. CM may be thus more recommended for studies where difficult kinematics measurement is required (e.g. free hand movement).

Another kinematics description was proposed by Giurintano et al., (1995) as the "virtual five linked segments" and may fix these problems. However, no anthropometric measurement of tendon locations was provided in the literature with this reference system and thus tendon tension computing is not possible.

As a limitation of Smutz model, it should be noted that the anthropometric data of Smutz et al. (1998) were not normalized by the subject hand size contrary to Chao et al. (1989). This point could be important since moment arm length of FPL at TMC (as an example) varied from $0.65 \mathrm{~cm}$ to $0.79 \mathrm{~cm}$ (near $18 \%$ ) with $\mathrm{CM}$ for a hand length varying from $18 \mathrm{~cm}$ to $22 \mathrm{~cm}$. Additionally, Smutz et al. (1998) published the values of the moment arms for given angular values of Flexion/Extension and Abduction/Adduction, the other degrees of freedom being in a neutral position. These moment arms thus could be slightly different for a combination of Flexion/Extension and Abduction/Adduction.

A limit of the present study concerned the method of comparison with EMG data as the EMG measurements were taken from a reference study and were not performed during the current experiment whereas the tested postures, forces and subjects were similar. Also, the uses of 

external kinematics markers always add imprecision even if high attention has been paid in their placement. In spite of these limits, this study brought new information about the performance of both models in regards to EMG data and kinematic error sensibility. To conclude, this study points out the necessity of new anthropometric measurements of thumb tendon location strongly related to a relevant and in vivo reproducible kinematic description (Fowler et al., 2001; Cheze et al., 2001). 


\section{References}

An, K.N. Ueba, Y. Chao, E.Y. Cooney, W.P. Linscheid, R.L., 1983. Tendon excursion and moment arm of index finger muscles. Journal of Biomechanics 16, 419-425.

Buchholz, B., 1992. Anthropometric data for describing the kinematics of the human hand. Ergonomics 35, 261-273.

Chao, E.Y. An, K.N. Cooney, W.P. Linscheid, R.L., 1989. Biomechanics of the hand. In: World scientific, Singapore, Chap. 1 and 4.

Cheze, L. Doriot, N. Eckert, M. Rumelhart, C. Comtet, J.J., 2001. In vivo cinematic study of the trapezometacarpal joint. Chirurgie de la main 20, 23-30.

Cooney, W. Lucca, M. Chao, E. Linscheid, R. 1981. The kinesiology of the thumb trapeziometacarpal joint. Journal of bone and joint Surgery, American volume 63, $1371-1381$.

Fowler, N.K. Nicol, A.C. Condon, B. Hadley, D. 2001. Method of determination of threedimensional index finger moment arms and tendon lines of action using high resolution MRI scans. Journal of Biomechanics 34, 791-797

Giurintano, D.J. Hollister, A.M. Buford, W.L. Thompson, D.E. Myers, L.M., 1995. A virtual five-link model of the thumb. Medical Engineering and Physics 17, 297-303.

Harding, D.C. Brandt, K.D. Hillberry, B.M., 1993. Finger joint force minimization in pianists using optimisation techniques. Journal of Biomechanics 26, 1403-1412.

Hollister, A. Buford, W.L. Myers, L.M. Giurintano, D.J. Novick, A., 1992. The axes of rotation of the thumb carpometacarpal joint. Journal of Orthopaedic Research 10, 454-460.

Kaufman, K.R. An, K.N. Litchy, W.J. Cooney, W.P. Chao, Y.S., 1999. In-vivo function of the thumb muscles. Clinical Biomechanics 14, 141-151. 
Sancho-Bru, J.L. Perez-Gonzalez, A. Vergara-Monedero, M. Giurintano, D.J., 2001. A 3-D dynamic model of human finger for studying free movements. Journal of Biomechanics 34, 1491-500.

Smutz, W. Kongsayreepong, A. Hughes, R. Niebur, G. Cooney, W. An, K.N., 1998. Mechanical advantage of the thumb muscles. Journal of Biomechanics 31, 565-570.

Valero-Cuevas, F. Johanson, M.E. Towles, J.D., 2003. Towards a realistic biomechanical model of the thumb : The choice of kinematic description may be more critical than the solution method or the variability/uncertainty of musculoskeletal parameters. Journal of Biomechanics 36, 1019-1030.

Vigouroux, L. Quaine, F. Colloud, F. Paclet, F. Moutet, F., 2008. Middle and ring fingers are more exposed to pulley rupture than index and little during sport-climbing: a biomechanical explanation. Clinical Biomechanics 23, 562-570. 
214 Table 1: Finger muscle tendons acting on the thumb joints. FPL: flexor pollicis longus; TET:

215 terminal extensor tendon; FPB: flexor pollicis brevis, APB: abductor pollicis brevis, ADPO:

216 adductor pollicis oblique head; ADPT: adductor pollicis tranverse head; EPL: extensor

217 pollicis longus; OPP: opponents pollicis; APL: abductor pollicis longus; EPB: extensor

218 pollicis brevis.

219

\begin{tabular}{cccc}
\hline \hline Joints & IP & MP & TMC \\
\hline \hline & FPL & FPL & FPL \\
& TET & FPB & FPB \\
Muscles & & APB & OPP \\
and & ADPO & APB \\
Tendons & ADPT & ADPO \\
& & EPL & ADPT \\
& & EPB & APL \\
& & EPL \\
& & EPB \\
\hline
\end{tabular}


Table 2: Mean differences between CM and SM model, statistical results of correlation between both model results and EMG data and mean error implicated by a $+/-5^{\circ}$ error of dorsal hand plane axes localisations. * indicates a significant correlation $(\mathrm{p}<0.05)$.

\begin{tabular}{cccccc}
\hline \hline Muscle & $\begin{array}{c}\text { Mean } \\
\text { difference (N) }\end{array}$ & $\begin{array}{c}\text { SM EMG } \\
\text { correlation }\end{array}$ & $\begin{array}{c}\text { CM EMG } \\
\text { correlation }\end{array}$ & $\begin{array}{c}\text { SM mean 5 } \\
\text { error (N) }\end{array}$ & $\begin{array}{c}\text { CM mean 5 }^{\circ} \\
\text { error (N) }\end{array}$ \\
\hline \hline FPL & $0.54 \pm 0.50$ & $\mathrm{r}=0.89 \mathrm{t}=4.75^{*}$ & $\mathrm{r}=0.75 \mathrm{t}=2.82 *$ & $0.61 \pm 1.06$ & $0.29 \pm 0.54$ \\
\hline FPB & $1.28 \pm 1.12$ & $\mathrm{r}=0.87 \mathrm{t}=4.24^{*}$ & $\mathrm{r}=0.91 \mathrm{t}=5.48^{*}$ & $0.47 \pm 0.98$ & $0.63 \pm 0.81$ \\
\hline OPP & $3.15 \pm 2.41$ & $\mathrm{r}=-0.07 \mathrm{t}=-0.18$ & $\mathrm{r}=0.44 \mathrm{t}=1.20$ & $1.05 \pm 2.37$ & $0.70 \pm 0.99$ \\
\hline APB & $0.70 \pm 0.50$ & $\mathrm{r}=0.86 \mathrm{t}=4.06^{*}$ & $\mathrm{r}=0.85 \mathrm{t}=3.97 *$ & $0.74 \pm 1.42$ & $0.32 \pm 0.67$ \\
\hline ADPt & $0.73 \pm 0.72$ & $\mathrm{r}=0.74 \mathrm{t}=2.67^{*}$ & $\mathrm{r}=0.51 \mathrm{t}=1.45$ & $0.73 \pm 1.34$ & $0.47 \pm 0.74$ \\
\hline ADPo & $1.73 \pm 1.95$ & & & $1.53 \pm 2.69$ & $0.82 \pm 1.39$ \\
\hline APL & $6.18 \pm 4.11$ & $\mathrm{r}=0.33 \mathrm{t}=0.85$ & $\mathrm{r}=-0.11 \mathrm{t}=-0.27$ & $3.35 \pm 4.15$ & $0.89 \pm 1.37$ \\
\hline EPL & $2.50 \pm 1.82$ & $\mathrm{r}=0.87 \mathrm{t}=4.38^{*}$ & $\mathrm{r}=0.88 \mathrm{t}=4.39 *$ & $2.02 \pm 2.42$ & $0.69 \pm 1.16$ \\
\hline EPB & $1.08 \pm 1.20$ & $\mathrm{r}=0.87 \mathrm{t}=4.24 *$ & $\mathrm{r}=0.87 \mathrm{t}=4.34^{*}$ & $0.87 \pm 1.79$ & $0.49 \pm 1.00$ \\
\hline
\end{tabular}




\section{Figure Captions}

Figure 1: Coordinate systems used to define joint angles, and joint moments. $\mathscr{R}_{\text {dhp }}$ was used for Smutz'model and $\mathbb{R}_{\mathrm{t}}$ was used for Chao's model to describe the TMC joint motion. $\mathscr{R}_{\mathrm{dhp}} \mathrm{X}$ axis was defined to be along the second and third metacarpals. $\mathscr{R}_{\mathrm{t}}$ was defined according to the locations proposed by Chao et al. (1989). $\mathbb{R}_{\mathrm{mc}}$ was used in both models to define MP joint motion and $\mathscr{R}_{\mathrm{pp}}$ was used to define IP motion. Black arrows indicate the direction of simulated forces applied to the middle of the distal phalanx. Palmar force (P) was applied perpendicular to the pulp of the thumb. Dorsal force (D) was applied perpendicular to the nail of the thumb. Medial (M) and Lateral (L) forces were applied perpendicular to the radial and ulnar sides of the thumb respectively. For clarity, dorsal-lateral, lateral-palmar, palmar-medial and medialdorsal force were not drawn.

Figure 2: Thumb tendon tensions $(\mathrm{N})$ provided by Smutz'model (black figures) and Chao's model (grey figures) during application of various directional fingertip forces (dorsal (D), dorsal-lateral (D-L), lateral (L), lateral-palmar (L-P), palmar (P), palmar-medial (P-M), medial (M), medial-dorsal (M-D)) with a key pinch. EMG results taken from Valero-Cuevas et al. (2003) are also presented in this figure and classified according to four levels (null: no radial line, little: one radial line is indicated in the corresponding force direction, median: a double radial line is drawn, high: a triple radial line is drawn).

Figure 3: Thumb tendon tensions $(\mathrm{N})$ provided by Smutz'model (black figures) and Chao's model (grey figures) during application of various directional fingertip forces (dorsal (D), dorsal-lateral (D-L), lateral (L), lateral-palmar (L-P), palmar (P), palmar-medial (P-M), medial (M), medial-dorsal (M-D)) with a pulp pinch. As in figure 2, EMG data taken from Valero-Cuevas et al. (2003) are presented in this figure and classified according to four levels (null: no radial line, little: one radial line is indicated in the corresponding force direction, median: a double radial line is drawn, high: a triple radial line is drawn). 
254 Figure 4: Moment arms lengths $(\mathrm{cm})$ at TMC joint observed with Smutz' model (black solid symbols) and Chao'model (hollow symbols) during key pinch. Moments arms of SM and CM were both expressed in dorsal hand plane reference system with Smutz' movement definition (flexion and adduction had positive values). 
$259 \quad$ Figure 1

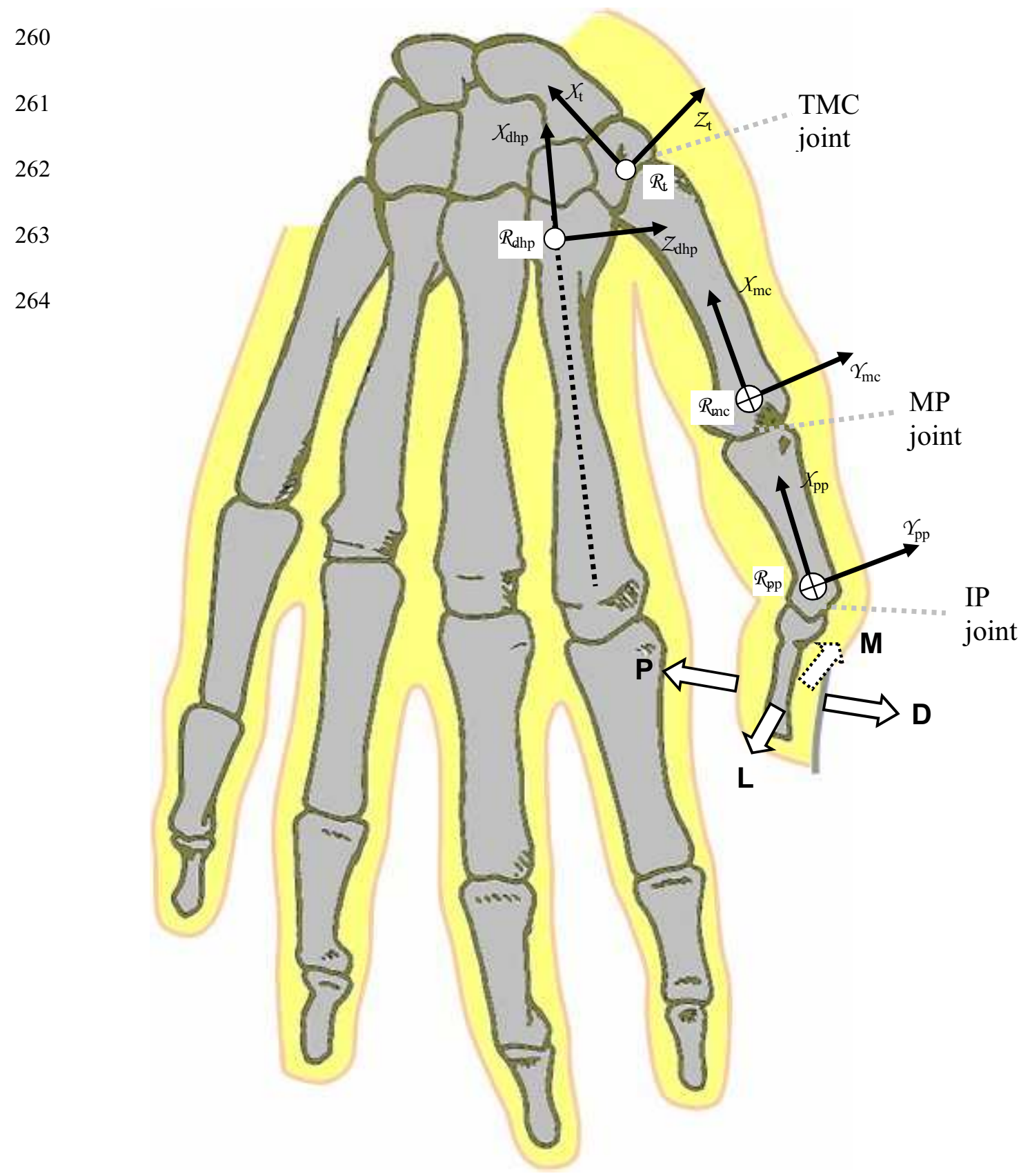


$\underline{\text { Figure } 2}$

Key pinch
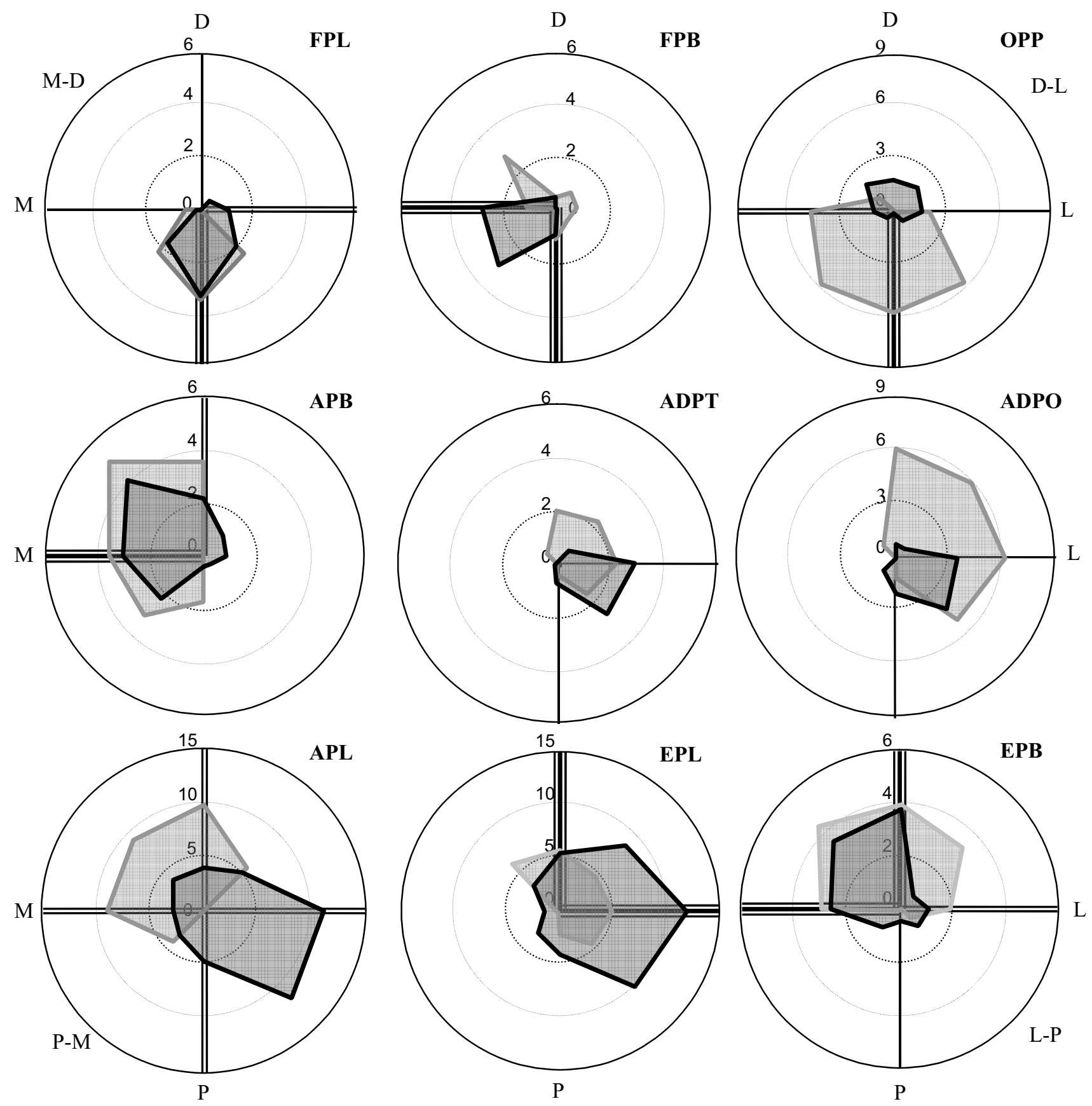
Figure 3

\section{Pulp pinch}

269
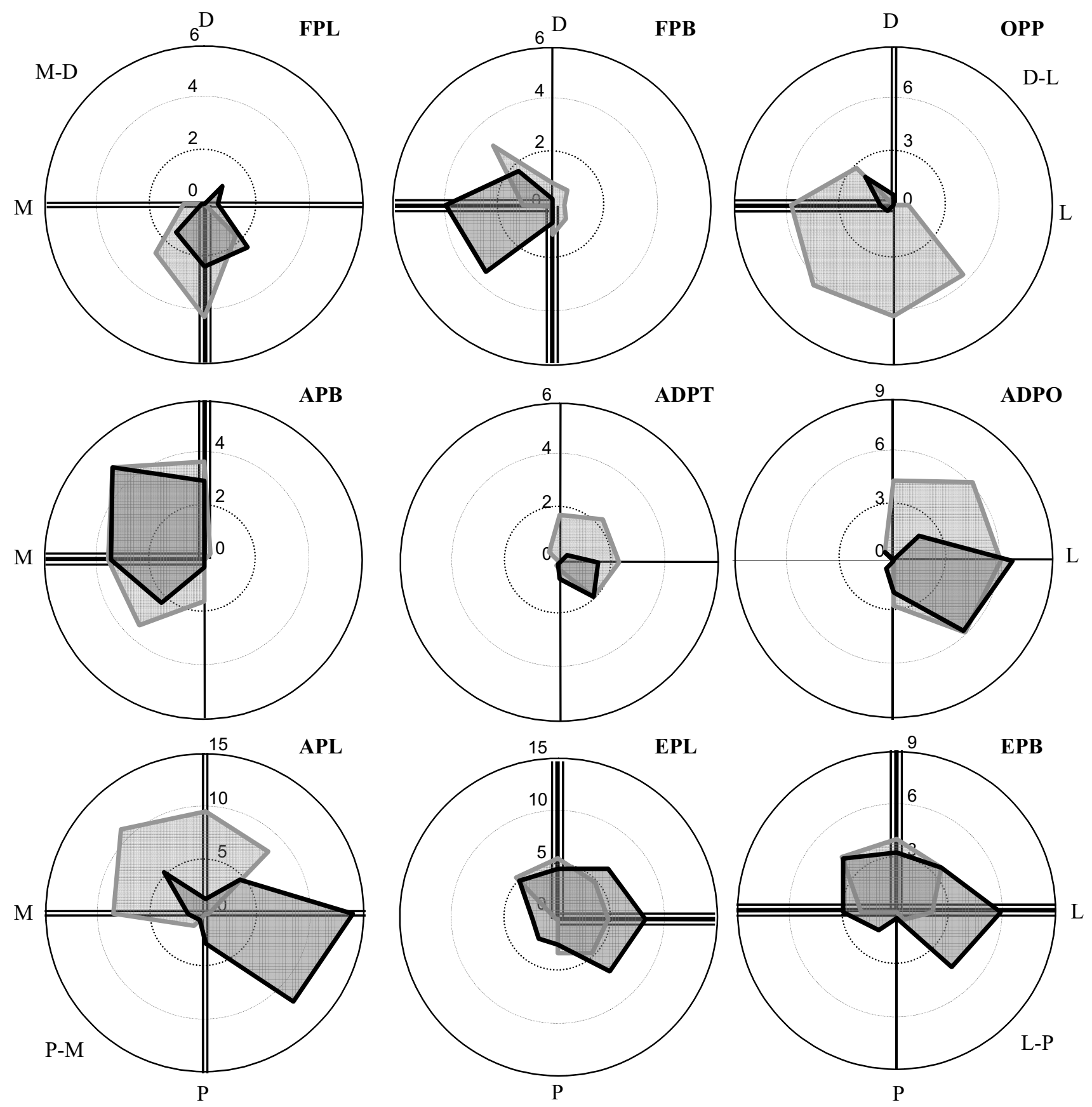
Figure 4:

271

272

273

\begin{tabular}{ccc}
$\mathrm{CM}$ & & $\mathrm{SM}$ \\
\hline $\boldsymbol{F}$ & $\mathrm{FPL}$ & $\bullet$ \\
$\bullet$ & $\mathrm{FPB}$ & $\boldsymbol{\bullet}$ \\
$\square$ & $\mathrm{OPP}$ & $\boldsymbol{\square}$ \\
$\diamond$ & $\mathrm{APB}$ & $\bullet$ \\
$\mathbf{8}$ & $\mathrm{ADPT}+\mathrm{O}$ & $\mathbf{z}$ \\
$\boldsymbol{\oplus}$ & $\mathrm{APL}$ & $\boldsymbol{\oplus}$ \\
$\Delta$ & $\mathrm{EPL}$ & $\boldsymbol{\Delta}$ \\
$\circ$ & $\mathrm{EPB}$ & $\bullet$
\end{tabular}

\section{Adduction}

3

2

$\mathbf{z}_{\mathrm{O}}$ $\mathbf{z}_{\mathrm{T}}$

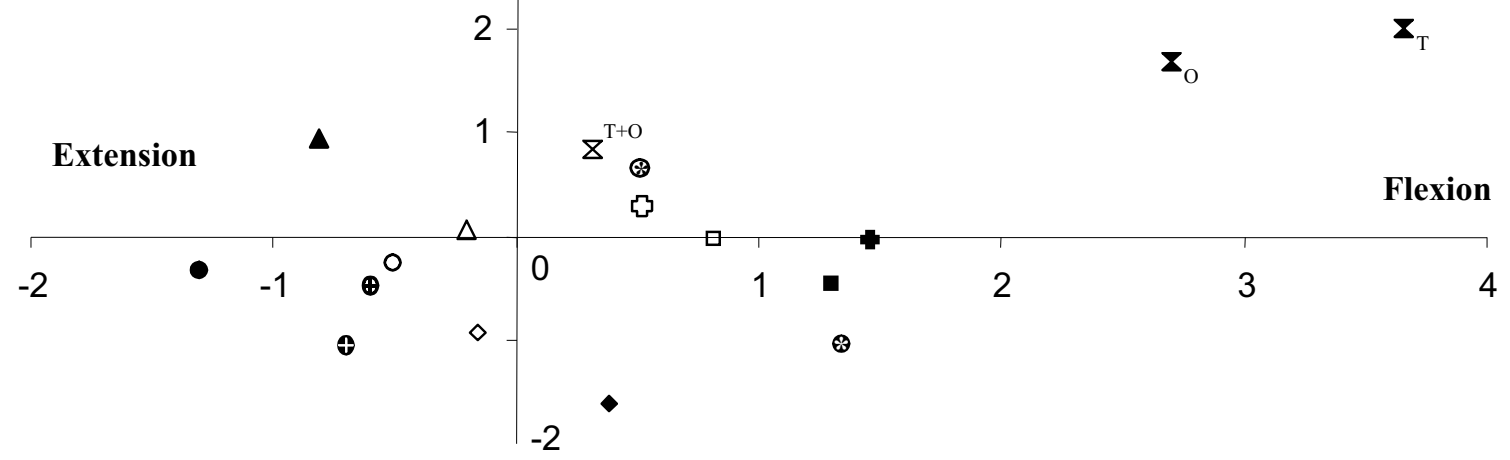

Abduction 